\title{
Mesenteric Phlebosclerosis Associated with Herbal Medicine
}

\author{
Kimitoshi Kubo ${ }^{a}$ Noriko Kimura $^{b} \quad$ Norishige Maiya $^{a}$ Soichiro Matsuda ${ }^{a}$ \\ Momoko Tsuda ${ }^{a}$ Mototsugu Kato ${ }^{a}$ \\ ${ }^{a}$ Department of Gastroenterology, National Hospital Organization Hakodate National \\ Hospital, Hakodate, Japan; 'bepartment of Pathology, National Hospital Organization \\ Hakodate National Hospital, Hakodate, Japan
}

\section{Keywords}

Mesenteric phlebosclerosis $\cdot$ Herbal medicine $\cdot$ Mesenteric vein calcification

\begin{abstract}
Mesenteric phlebosclerosis (MP) associated with herbal medicine is rarely reported and its endoscopic and radiological features remain poorly described. An 81-year-old woman was admitted to our hospital for right lower abdominal pain and high-grade fever. Computed tomography $(\mathrm{CT})$ revealed wall thickening, pericolic fat stranding, and linear calcifications extending from the cecum to the hepatic flexure of the colon. Ultrasonography (US) revealed wall thickening of the cecum and ascending colon. Colonoscopy (CS) revealed dark-purple edematous mucosa with erosion and ulcers from the cecum to the hepatic flexure of the colon. The patient was histopathologically diagnosed with MP, discontinued orengedokuto, and was treated with intravenous antibiotics for 1 week. Six months after treatment, US and CT revealed no significant changes, but CS showed improvement in dark-purple edematous mucosa with erosion and ulcers. To our knowledge, this report represents a valuable addition to the MP literature describing a rare case of MP associated with herbal medicine.
\end{abstract}




\section{Case Reports in Gastroenterology}

\begin{tabular}{l|l}
\hline Case Rep Gastroenterol 2020;14:516-521 \\
\hline DOI: 10.1159/000508931 & $\begin{array}{l}\text { @ 2020 The Author(s). Published by S. Karger AG, Basel } \\
\text { www.karger.com/crg }\end{array}$ \\
\hline
\end{tabular}

Kubo et al.: Mesenteric Phlebosclerosis Associated with Herbal Medicine

\section{Introduction}

Mesenteric phlebosclerosis (MP) is a chronic ischemic disease caused by venous return impairment associated with fibrous thickening and calcification of mesenteric veins [1]. Since it was first reported in Japan by Koyama et al. [2] as "chronic ischemic colitis causing stenosis," reports of MP followed mainly from Japan and other Asian countries. Yoshinaga et al. [3] reported that the prevalence rate in Japan was 0.10 per 100,000 population, with the mean age being 61.8 years and the male/female ratio being 2:3. Recently, a relationship between MP and the use of herbal medicine containing gardenia fruit (e.g., geniposide) was suggested in a large-scale nationwide survey in Japan [4].

MP associated with herbal medicine is rare, and its pre- and posttreatment endoscopic and radiological features remain largely unclear. We herein report a case of MP associated with herbal medicine.

\section{Case Presentation}

An 81-year-old woman was admitted to our hospital for right lower abdominal pain and high-grade fever. Of note, she had a history of hypertension and had taken orengedokuto (T)15), a Chinese herbal medicine, for 7.4 years for her hypertension-related symptoms. Physical examination findings included: height, $152 \mathrm{~cm}$; weight, $53 \mathrm{~kg}$; clear consciousness; blood pressure, $153 / 89 \mathrm{~mm} \mathrm{Hg}$; heart rate, $66 / \mathrm{min}$; and body temperature, $38.2^{\circ} \mathrm{C}$. Laboratory findings included: white blood cell count, $11.3 \times 10^{9} / \mathrm{L}$; and C-reactive protein, $2.1 \mathrm{mg} / \mathrm{dL}$. Computed tomography (CT) revealed wall thickening, pericolic fat stranding, and linear calcifications extending from the cecum to the hepatic flexure of the colon (Fig. 1a-c). Ultrasonography (US) revealed wall thickening of the cecum (Fig. 2a) and ascending colon (Fig. 2b). Colonoscopy (CS) revealed dark-purple edematous mucosa with erosion and ulcers from the cecum to the hepatic flexure of the colon (Fig. 3a-c). Histopathological examination of the biopsy specimens showed remarkable hyalinization of small vessels and chronic inflammatory cell infiltration in the mucosa (Fig. 3g, h). Elastica-Masson trichrome stain revealed deposition of hyalinized collagen fibers, which led to amyloidosis being ruled out (Fig. 3i).

The patient was diagnosed with MP, discontinued orengedokuto, and was treated with intravenous antibiotics for 1 week. She was discharged on day 12 of hospitalization because of improvement in abdominal pain and fever. No abdominal pain or fever was observed after discharge. Six months after discontinuation of orengedokuto, follow-up examinations were performed. CT revealed disappearance of pericolic fat stranding, but no significant change in wall thickening or linear calcifications (Fig. 1d-f); US revealed wall thickening similar to that seen in the initial US examination (Fig. 2c, d); and CS revealed improvement of the edema as well as disappearance of the erosion and ulcers, with the dark-purple mucosa becoming brighter (Fig. 3d-f).

\section{Discussion and Conclusion}

Our case offers two important clinical implications. First, long-term use of herbal medicine may be associated with the onset of MP. Further, MP associated with herbal medicine is rare and its pre- and posttreatment endoscopic and radiological features remain largely unclear. 


\section{Case Reports in Gastroenterology}

\begin{tabular}{l|l}
\hline Case Rep Gastroenterol 2020;14:516-521 \\
\hline DOI: 10.1159/000508931 & $\begin{array}{l}\text { @ 2020 The Author(s). Published by S. Karger AG, Basel } \\
\text { www.karger.com/crg }\end{array}$ \\
\hline
\end{tabular}

Kubo et al.: Mesenteric Phlebosclerosis Associated with Herbal Medicine

MP is a disease that causes symptoms such as abdominal pain, diarrhea, constipation, or ileus due to chronic ischemic changes in the superior mesenteric vein [5]. Previously, an association was reported between MP and portal hypertension, blood coagulation disorders, CREST syndrome, dialysis, vasculitis, diabetes mellitus, hyperlipidemia, or hypertension [6]. Recently, MP has been shown to be associated with the use of herbal medicine containing geniposide [4-8], an iridoid glucoside, which is a major constituent of gardenia fruit and is widely used as a component of herbal medicine [9]. Orally administered, geniposide is transformed by $\beta$-glucosidase produced by the intestinal flora of the proximal colon to genipin [10], a cross-linking reagent and a natural dye. As a potential mechanism of onset of MP, genipin is thought to be involved in thickening of collagen fibers in the vein by forming intermolecular collagen cross-links, while at the same time contributing to a dark-purple-colored colonic mucosa through its natural reaction with amino acids and proteins [8]. It is reported, however, that herbal medicine is used for a long period of time, with the mean duration of its use being 13.6 years [4], 11.1 years [6], and 10.7 years [7], and that the cumulative dose of gardenia fruit in excess of some 5,000 $\mathrm{g}$ contributes to and/or accelerates the development of MP [6]. Thus, our patient is of particular interest in that she had taken orengedokuto for 7.4 years, which amounted to a cumulative dose of about 5,400 g of geniposide.

The second implication is that our patient was evaluated for MP using multiple modalities (CT, US, and CS) before and after treatment, despite a paucity of US findings of MP. While it is reported that CT findings of MP typically include colonic wall thickening and venous calcifications of the right hemicolon [11] and endoscopic findings on MP include abnormal coloration (dark purple or bronze) with reduced distensibility, erosion/ulcers, or stenosis of the right hemicolon [4], these characteristic image findings were also recognized in our patient. In addition, while US did not allow for visualization of venous calcifications, it did allow for visualization of colonic wall thickening of the right hemicolon. Interestingly, 6 months after discontinuation of treatment (orengedokuto), US and CT revealed no significant changes, but CS showed improvement in the dark-purple edematous mucosa with erosion and ulcers, in agreement with the report of Miwa et al. [12] showing that the endoscopic findings improved after treatment but the CT findings did not. Further accumulation of data is needed from longterm posttreatment follow-up of patients with MP to verify these findings.

In conclusion, long-term use of herbal medicine may be associated with the onset of MP, suggesting that clinicians should keep this disease in mind as a potential complication. In addition, not only CS and CT, but also US as a noninvasive modality, may prove helpful as followup imaging examinations for patients with MP.

\section{Statement of Ethics}

Written informed consent was obtained from the patient for publication of this case report and any accompanying images.

\section{Conflict of Interest Statement}

The authors have no conflicts of interest to disclose in association with this study. 


\section{Case Reports in Gastroenterology}

\section{Funding Sources}

No funding was obtained for this study.

\section{Author Contributions}

K. Kubo, N. Kimura, N. Maiya, S. Matsuda, M. Tsuda, and M. Kato carried out and confirmed the diagnosis, provided the details of the case, and contributed to the design of the report. K. Kubo and M. Kato drafted the manuscript. All authors have read and approved the final version of the manuscript.

\section{References}

1 Iwashita A, Yao T, Schlemper RJ, Kuwano Y, Yao T, Iida M, et al. Mesenteric phlebosclerosis: a new disease entity causing ischemic colitis. Dis Colon Rectum. 2003 Feb;46(2):209-20.

2 Koyama N, Koyama H, Hanajima T, Matsubara N, Fujisaki J, Shimoda T. Chronic ischemic colitis causing stenosis, report of a case. Stomach Intestine. 1991;26:455-60. Japanese.

3 Yoshinaga S, Nakamura K, Harada N, Yao T. Clinical features of idiopathic mesenteric phlebosclerosis. Stomach Intestine. 2009;44:163-9. Japanese.

4 Shimizu S, Kobayashi T, Tomioka H, Ohtsu K, Matsui T, Hibi T. Involvement of herbal medicine as a cause of mesenteric phlebosclerosis: results from a large-scale nationwide survey. J Gastroenterol. 2017 Mar;52(3): 308-14.

5 Minami K, Fujiie M, Fushimi F, Taguchi K, Nobutoh Y, Yoshida D, et al. Ascending colon cancer coincident with mesenteric phlebosclerosis associated with the long-term oral intake of Chinese herb containing gardenia fruit: A case report and literature review. Int Cancer Conf J. 2017 Feb;6(2):70-5.

6 Nagata Y, Watanabe T, Nagasaka K, Yamada M, Murai M, Takeuchi S, et al. Total dosage of gardenia fruit used by patients with mesenteric phlebosclerosis. BMC Complement Altern Med. 2016 Jul;16(1):207.

7 Nagata Y, Watanabe T, Nagasaka K, Yamada M, Saito M, Yazaki T, et al. Clinical search for undiagnosed mesenteric phlebosclerosis at outpatient departments specializing in herbal (kampo) medicine. Intern Med. 2016;55(6):573-81.

8 Hiramatsu K, Sakata H, Horita Y, Orita N, Kida A, Mizukami A, et al. Mesenteric phlebosclerosis associated with long-term oral intake of geniposide, an ingredient of herbal medicine. Aliment Pharmacol Ther. 2012 Sep;36(6):575-86.

9 Hou YC, Tsai SY, Lai PY, Chen YS, Chao PD. Metabolism and pharmacokinetics of genipin and geniposide in rats. Food Chem Toxicol. 2008 Aug;46(8):2764-9.

10 Akao T, Kobashi K, Aburada M. Enzymic studies on the animal and intestinal bacterial metabolism of geniposide. Biol Pharm Bull. 1994 Dec;17(12):1573-6.

11 Yao T, Iwashita A, Hoashi T, Matsui T, Sakurai T, Arima S, et al. Phlebosclerotic colitis: value of radiography in diagnosis - report of three cases. Radiology. 2000 Jan;214(1):188-92.

12 Miwa W, Hiratsuka T, Kamiya Y, Shoji H, Tei S, et al. Improved mesenteric phlebosclerosis after cessation of Chinese herbal medicine, a case report with an observation period of 7 years from onset. Gastroenterol Endosc. 2015;57:1278-83. Japanese. 
Case Reports in Gastroenterology

\begin{tabular}{l|l}
\hline Case Rep Gastroenterol 2020;14:516-521 \\
\hline DOI: 10.1159/000508931 & $\begin{array}{l}\text { @ 2020 The Author(s). Published by S. Karger AG, Basel } \\
\text { www.karger.com/crg }\end{array}$ \\
\hline
\end{tabular}

Kubo et al.: Mesenteric Phlebosclerosis Associated with Herbal Medicine
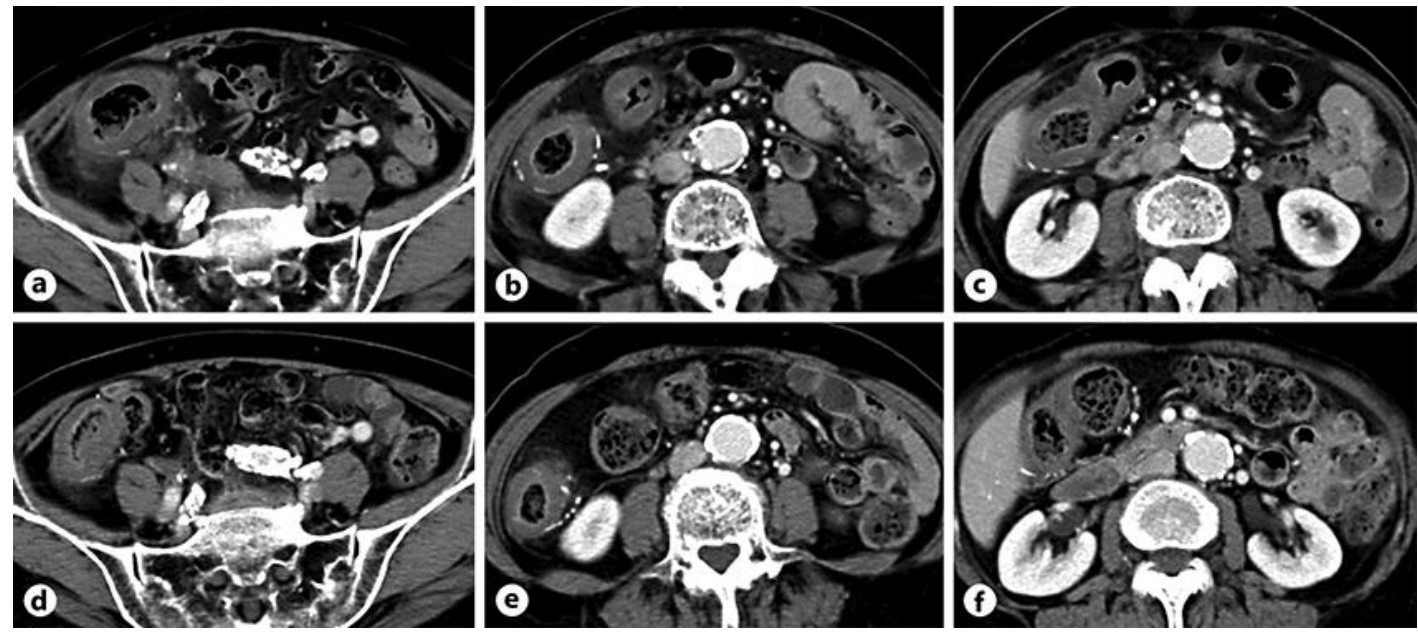

Fig. 1. a-c CT on admission revealed wall thickening, pericolic fat stranding, and linear calcifications extending from the cecum to the hepatic flexure of the colon. $d-f$ CT performed 6 months after treatment revealed disappearance of pericolic fat stranding, but no significant change in wall thickening or linear calcifications. CT, computed tomography.

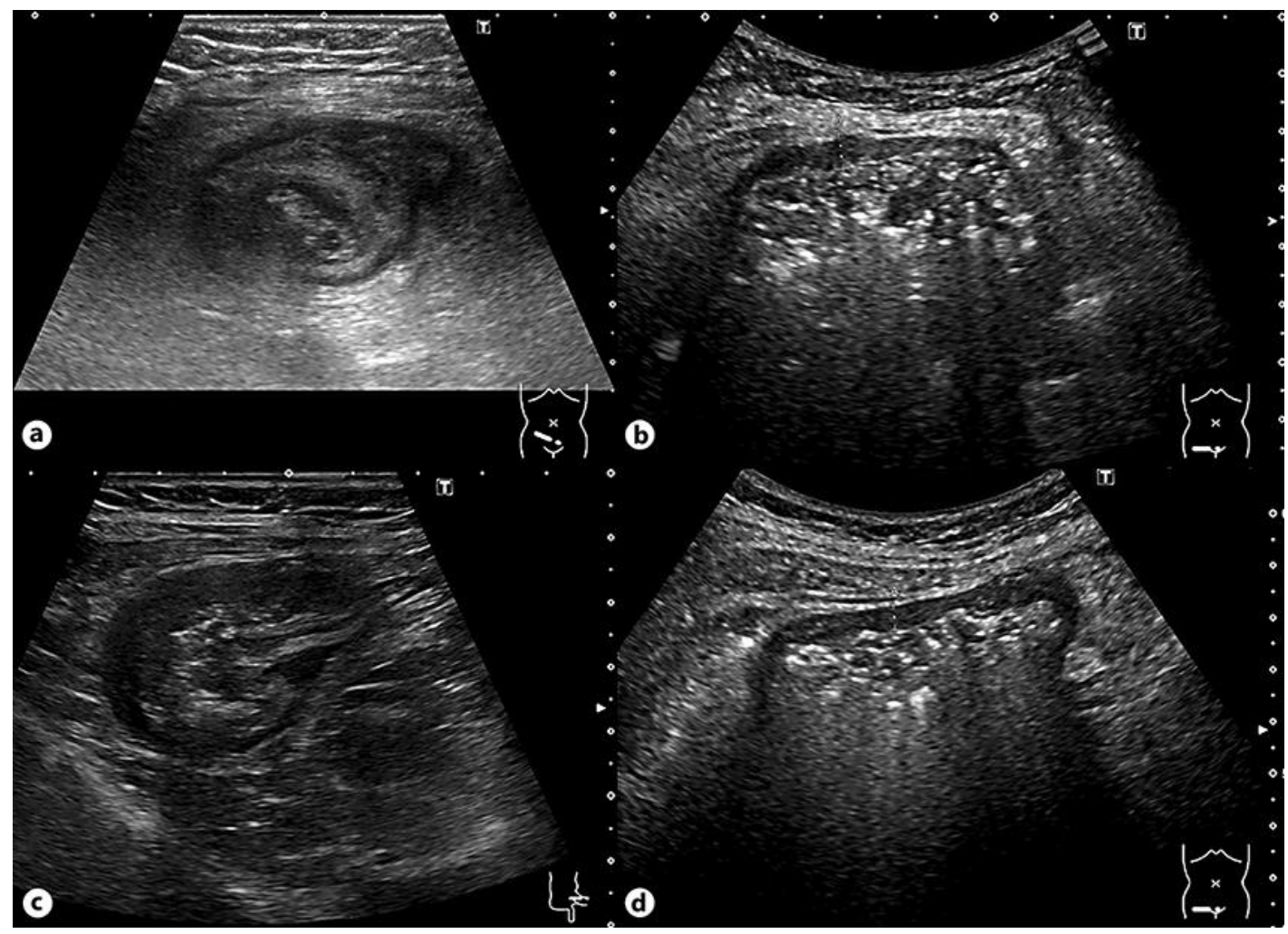

Fig. 2. US revealed wall thickening of the cecum (a) and ascending colon (b). US performed 6 months after treatment revealed wall thickening similar to that in the initial US (c, d). US, ultrasonography. 


\section{Case Reports in Gastroenterology}
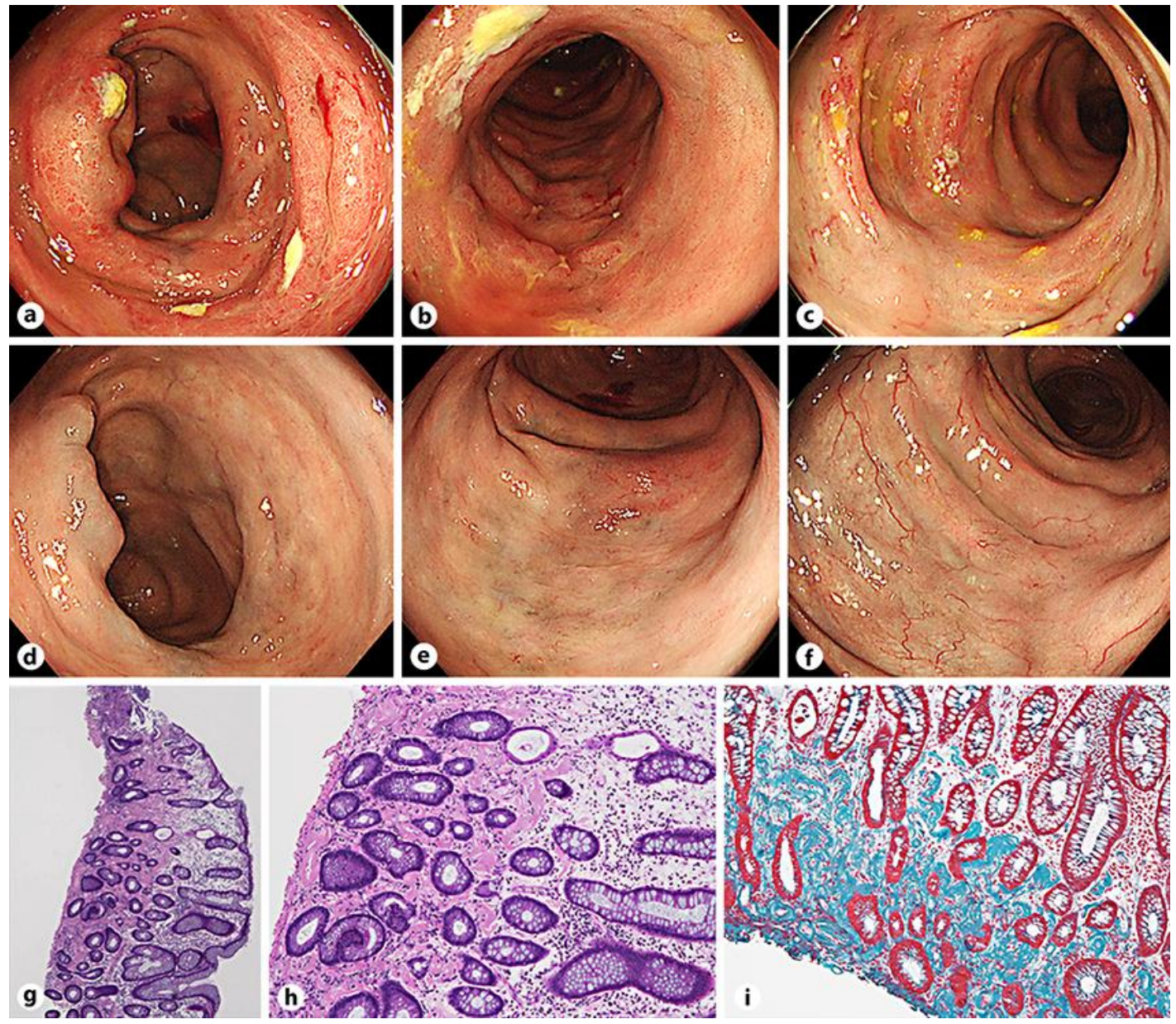

Fig. 3. Endoscopic findings and histopathological examination of the biopsy specimens. a-c CS revealed dark-purple edematous mucosa with erosion and ulcers from the cecum to the hepatic flexure of the colon. d-f CS performed 6 months after treatment revealed improvement of edema and disappearance of erosion and ulcers, with the dark-purple mucosa becoming brighter. Histopathological examination of the biopsy specimens showed remarkable hyalinization of small vessels and chronic inflammatory cell infiltration in the mucosa $(\mathbf{g}, \times 20 ; \mathbf{h}, \times 40)$. $\mathbf{i}$ Elastica-Masson trichrome stain revealed deposition of hyalinized collagen fibers, which led to amyloidosis being ruled out $(\times 40)$. CS, colonoscopy. 